\title{
Incorporating temporal distribution of population- level viral load enables real-time estimation of COVID-19 transmissibility
}

Yun Lin

University of Hong Kong

Bingyi Yang

HKU https://orcid.org/0000-0002-0811-8332

Sarah Cobey

University of Chicago https://orcid.org/0000-0001-5298-8979

Eric Lau

University of Hong Kong https://orcid.org/0000-0002-6688-9637

\section{Dillon Adam}

The University of Hong Kong

Jessica Wong

The University of Hong Kong

Helen Bond

University of Hong Kong

Justin K. Cheung

University of Hong Kong

Faith Ho

The University of Hong Kong

Huizhi Gao

The University of Hong Kong

Sheikh Taslim Ali

The University of Hong Kong https://orcid.org/0000-0002-8631-9076

Nancy Leung

University of Hong Kong https://orcid.org/0000-0001-7314-840X

Tim Tsang

University of Hong Kong

Peng Wu

The University of Hong Kong https://orcid.org/0000-0003-1157-9401

\section{Gabriel Leung}

School of Public Health, LKS Faculty of Medicine, The University of Hong Kong https://orcid.org/00000002-2503-6283 
Benjamin Cowling ( $\nabla$ bcowling@hku.hk)

University of Hong Kong https://orcid.org/0000-0002-6297-7154

\section{Article}

Keywords: COVID-19, viral load, epidemic dynamics

Posted Date: August 27th, 2021

DOl: https://doi.org/10.21203/rs.3.rs-841953/v1

License: (c) (1) This work is licensed under a Creative Commons Attribution 4.0 International License. Read Full License

Version of Record: A version of this preprint was published at Nature Communications on March 3rd, 2022. See the published version at https://doi.org/10.1038/s41467-022-28812-9. 
1 Incorporating temporal distribution of population-level viral load enables real-

2 time estimation of COVID-19 transmissibility

3 Yun Lin ${ }^{1, *}$, Bingyi Yang1,*, Sarah Cobey ${ }^{2}$, Eric H. Y. Lau ${ }^{1,3}$, Dillon C. Adam ${ }^{1}$, Jessica Y.

4 Wong $^{1}$, Helen S. Bond ${ }^{1}$, Justin K. Cheung ${ }^{1}$, Faith Ho ${ }^{1}$, Huizhi Gao ${ }^{1}$, Sheikh Taslim Ali' ${ }^{1,3}$,

5 Nancy H. L. Leung1, Tim K. Tsang1, Peng Wu ${ }^{1,3}$, Gabriel M. Leung1,3, Benjamin J.

$6 \quad$ Cowling1,3

8 Affiliations:

1. WHO Collaborating Centre for Infectious Disease Epidemiology and Control,

*Joint first authors with equal contribution

\section{Corresponding author:}

19 Benjamin J. Cowling, School of Public Health, Li Ka Shing Faculty of Medicine, The

20 University of Hong Kong, 7 Sassoon Road, Pokfulam, Hong Kong

21 Tel: +852 3917 6711; Email: bcowling@hku.hk

23 Word count (abstract): 149

24. Word count (main text): 2069 


\section{Abstract}

27 Many locations around the world have used real-time estimates of the time-varying

28 effective reproductive number $\left(R_{t}\right)$ of COVID-19 to provide evidence of transmission

29 intensity to inform control strategies. Estimates of $R_{t}$ are typically based on statistical

30 models applied to case counts and typically suffer lags of more than a week because of

31 the incubation period and reporting delays. Noting that viral loads tend to decline over

32 time since illness onset, analysis of the distribution of viral loads among confirmed

33 cases can provide insights into epidemic trajectory. Here, we analyzed viral load data on

34 confirmed cases during two local epidemics in Hong Kong, identifying a strong

35 correlation between temporal changes in the distribution of viral loads (measured by

36 cycle threshold values) and estimates of $R_{t}$ based on case counts. We demonstrate that

37 cycle threshold values could be used to improve real-time $R_{t}$ estimation, enabling more

38 timely tracking of epidemic dynamics. 


\section{Introduction}

41 Monitoring the transmissibility of an emerging infectious disease in a timely manner is crucial to monitor the effectiveness of public health and social measures and to inform better control policies. During the coronavirus diseases 2019 (COVID-19) pandemic, real-time assessment of transmissibility has generally been achieved through monitoring the time-varying effective reproductive number, $R_{t}$. A number of statistical approaches have been developed to allow estimation of $R_{t}$ from time series of daily case counts either recorded by date of illness onset or by date of laboratory confirmation ${ }^{1}$. However, these approaches tend only to be able to estimate $R_{t}$ with a lag of one week or more, because COVID-19 transmission can occur prior to illness onset ${ }^{2}$, because of

50 delays between individuals being infected and PCR detectable and/or showing symptoms (which are typically around 3-5 days for COVID-19) ${ }^{3}$, and because of delays between illness onset and diagnosis. In Hong Kong, we estimated $R_{t}$ with a 7-day lag by accounting for pre-symptomatic transmission and reporting delays ${ }^{4,5}$.

An individual infected by SARS-CoV-2 will typically experience viral load peaking around illness onset and monotonically decreasing during the following two weeks ${ }^{6}$. While viral loads can vary across individuals, with some shedding more than others ${ }^{7}$, the mean distribution of viral loads from a group of patients measured around the time 59 of illness onset will tend to have higher values than that of viral loads from a group of 60 patients measured at a later time after infection ${ }^{8}$. Collectively, higher population-level 61 viral loads would correlate with more infected persons being earlier in the course of 62 infection and vice versa ${ }^{9}$. Viral loads can be proxied by cycle threshold (Ct) values in the real-time quantitative reverse-transcription polymerase chain reaction (RT-PCR) assay, 64 with lower Ct values indicative of higher viral loads. 
66 A recent study showed that the distribution of viral loads among confirmed cases can

67 provide inferences on transmission dynamics within populations, where population-

68 level Ct values skewing towards lower values indicate more individuals have been

69 recently infected, corresponding to an increasing rate of epidemic growth in the

70 community especially where single strain dominates ${ }^{9}$. The method was demonstrated in

71 a modelling study using cross-sectional samples in a small-scale outbreak in Boston ${ }^{9}$

72 while the correlation was also observed over an epidemic wave elsewhere ${ }^{10}$. Here, we

73 incorporated Ct values from COVID-19 cases in Hong Kong, a location with intense

74. surveillance and case-finding efforts, to determine whether inclusion of data on Ct

75 values could allow real-time estimation of transmissibility.

\section{$77 \quad$ Results}

78 In Hong Kong, COVID-19 cases are detected through clinical diagnosis for individuals

79 with acute respiratory symptoms and public health surveillance for the community with

80 a priority to people with pre-defined high exposure risks ${ }^{11}$ (see Methods). Close

81 contacts of confirmed cases are traced and placed into quarantine outside the home,

82 and repeatedly tested. All laboratory-confirmed COVID-19 cases, including

83 asymptomatic cases, are isolated within hospital and receive multiple RT-PCR tests

84 during their stay. After excluding imported cases, we analyzed the first available record

85 of Ct value for each confirmed case and characterized the daily distribution of Ct values

86 (measured by mean and skewness) that were sorted by sampling days. We included two

87 consecutive epidemics in July-August 2020 (i.e., the third wave) and November 2020

88 through March 2021 (i.e., the fourth wave), which were dominated by local

89 transmissions instead of imported cases $^{5,12}$. Ct values were available for $96 \%$ (8268 out 
90 of 8646) of local cases that were confirmed during the study period. The local COVID-19

91 vaccination program began in late February 2021 towards the end of the period

92 studied. Variants of concerns (VoCs) were not reported among local cases during the

93 study period, while two separate wild-type lineages dominated the two studied waves ${ }^{13}$

We first examined the correlation between the distribution of daily Ct values and the local transmission dynamics 5 (measured by the incidence-based $R_{t}$; see Methods). The temporal Ct distribution tracked very closely the incidence-based $R_{t}$ over the two epidemic waves (Fig. 1; Supplementary Fig. 1). Higher values of incidence-based $R_{t}$ were found when the average Ct values decreased (Spearman's correlation coefficient, $\rho$ $=-0.79, \mathrm{P}<0.001$ for the third wave and $\rho=-0.52, \mathrm{P}<0.001$ for the fourth wave) and when the Ct skewed towards lower values (i.e., greater values of skewness estimates; $\rho$

$102=0.80, \mathrm{P}<0.001$ for the third wave and $\rho=0.27, \mathrm{P}<0.001$ for the fourth wave) (Fig. 1; 103 Supplementary Table 1).

To confirm that the changes in the observed daily Ct distribution were mostly driven by 106 the epidemic dynamics despite individual variations in viral shedding, we extrapolated 107 the Ct value back to illness onset for symptomatic cases by assuming that log108 transformed Ct values increase after illness onset (see Methods). The skewness of the expected population-level Ct value distribution at illness onset (coefficient of variation:

110 0.37) was less variable than that of the observation at time of sampling (coefficient of 111 variation: 0.80 ), suggesting a relatively stable peaking level of viral loads across 112 individuals over the course of the epidemic (Supplementary Fig. 2). 
114 To use Ct values for real-time assessing COVID-19 transmissibility in the community, we

115 fitted a log-linear regression to daily incidence-based $R_{t}$ on daily mean and skewness of

116 Ct values at sampling during the third wave (i.e., training period; see Methods). We

117 found that the distribution of Ct values explained $72 \%$ of the observed variations in

118 incidence-based $R_{t}$ during the training period (Supplementary Table 2). We then

119 applied the trained model to the daily Ct distributions in the fourth wave (i.e., testing

120 period) to estimate $R_{t}$ in real time (i.e., Ct-based $R_{t}$ ). We found that the Ct-based

121 method provided accurate real-time estimations of $R_{t}$ during the 7-day lagged window

122 suffered by the conventional incidence-based $R_{t}$ estimation method (Fig. 2a). We found

123 high correlations between Ct- and incidence-based $R_{t}$ for both training (Spearman's

124 correlation coefficient, $\rho=0.81, \mathrm{P}<0.001)$ and testing periods $(\rho=0.48, \mathrm{P}<0.001)$ (Fig.

125 2b-d). We conducted a sensitivity analysis to account for the potential impact of age on

126 Ct distributions and therefore the Ct-based $R_{t}$, and found that the high correlation

127 between Ct- and incidence-based $R_{t}$ remained (Supplementary Fig. 3).

128

129 We performed a further validation of our results by training the model using data from

130 November to December 2020 and predicting the third wave and found the high

131 accuracy of predictions still held (Supplementary Fig. 4). We also performed a 10-fold

132 cross-validation, in which we randomly assigned data between 6 July 2020 to 31 March

1332021 into 10 validation sets. We found that on average $81 \%$ (ranging from $75 \%$ to $85 \%$ )

134 of the Ct- and incidence-based $R_{t}$ estimates were consistent (i.e., simultaneously below

135 or above 1) across validation sets. These results suggested that relationships between

136 Ct distributions and $R_{t}$ estimates were not affected by temporal autocorrelation of

137 incidence-based $R_{t}$. In addition, we found that our model predictions were not impacted

138 by the length and timing of the training period as long as the training period covered 
139 one complete epidemic wave (Supplementary Fig. 5). The key element of a suitable

140 training period is to cover a period with sufficient samples to reflect epidemic changes,

141 which usually starts from 2-3 weeks before the case peak till around 1-2 weeks after the

142 peak (Supplementary Fig. 5). Longer training periods did not necessarily lead to better

143 performance, possibly due to the variability in the longer tail with low numbers of

144 samples (Supplementary Fig. 1 and 5).

\section{Discussion}

147 In this study, we applied a simplified Ct-based method to provide precise estimates of 148 daily $R_{t}$ and demonstrated that such a method could be used for real-time $R_{t}$

149 estimation. Conventionally, the main challenge in estimating $R_{t}$ in real-time was largely 150 caused by the delays between an individual being infected and being PCR detectable or 151 illness onset ${ }^{3,14}$. Linking the incidence-based $R_{t}$ and the population-level Ct distribution 152 among samples collected on a given day was able to mitigate the right-censoring issue 153 (i.e., missing cases that were infected but not-yet-observed due to the latent period) 154 encountered by incidence-based methods ${ }^{1,3}$. The few studies that have used population 155 viral loads to infer COVID-19 epidemics only provided probability distributions of the 156 estimated position of a community within an epidemic curve ${ }^{9,15}$, while our method 157 provides precise longitudinal $R_{t}$ estimates, which further demonstrates the potential to 158 improve real-time situational awareness using the Ct-based methods. In addition, we 159 showed that the daily $\mathrm{Ct}$ distribution could be applied for tracking epidemics under a 160 symptom and contact-tracing based setting, such as Hong Kong, providing empirical 161 data to support the hypotheses generated from previous Ct-based studies 9 . 
163 Our simplified Ct-based method also provides an approach for real-time estimation of

164 $R_{t}$ without requiring intensive surveillance of COVID-19 (i.e., accurate daily case counts

165 by onset or diagnosis), which is of great significance especially for areas and time

166 periods with limited surveillance capacity. Our results showed that population-level Ct

167 distributions remained informative in tracking epidemic changes over time regardless

168 of changes in surveillance in Hong Kong, especially by expanding the testing capacity

169 during the fourth wave in our case ${ }^{11}$. As the main prerequisite of the model was the

170 distribution of Ct values among confirmed cases, our findings were less sensitive to

171 changes in case reporting (e.g., due to definition changes and/or testing capacity

172 constraints), which, by contrast, would greatly affect conventional incidence-based $R_{t}$

173 estimation ${ }^{16,17}$. For example, our Ct-based $R_{t}$ indicated sustained community

174 transmissions during the period between late October to early November 2020. This

175 result is supported by $R_{t}$ estimates generated from genomic data, which determined

176 that SARS-CoV-2 introductions in September 2020, otherwise missed by incidence-

177 based $R_{t}$ methods, seeded undetected and sustained chains of transmission that

178 eventuated in the fourth wave expansion detected in early November $2020^{13}$. Of note,

$179 R_{t}$ estimated with very few Ct samples (e.g., less than five) collected on a given day can

180 lead to larger uncertainty, though we believe this may not be an issue for most areas

181 with prevalent local COVID-19 transmissions, even if testing capacity is limited.

183 Our work is not the first attempt to improve real-time tracking COVID-19

184 transmissibility. Another study ${ }^{18}$ demonstrated that using sewage surveillance could

185 shorten the prediction delays to two days ahead test positives, which, however, did not

186 fully overcome the delays between being infected and being PCR detectable ${ }^{3}$. In

187 addition, chances to locate sewage containing viral RNA could be low, especially when 
188 transmission in the community was low. Under such situation, our method, which

189 leverages existing information from confirmed cases, may be less resource-consuming

190 and faster to implement, as long as the reporting delay could be shortened.

192 Future applications of our method may need adaptation to different populations,

193 especially among those with which viral load trajectories differ. In particular,

194 populations with higher SARS-CoV-2 vaccination rates may expect increased average Ct

195 values when $R_{t}$ is greater than 1, as lower viral loads were found in cases who had

196 received COVID-19 vaccinations ${ }^{19}$. Similarly, increased Ct values when $R_{t}$ is greater than

1971 may also be found in populations younger than Hong Kong's population, due to the

198 generally lower viral loads observed among young people ${ }^{6}$. As such, while we believe

199 the intrinsic relationship between population viral loads and $R_{t}$ estimates will remain

200 valid (as long as the time relation between infection and viral shedding still holds),

201 recalibrations of the model may be needed when applying our model to different

202 populations.

204 By the time this study was performed, there were limited VoCs circulating in Hong 205 Kong $^{13}$, therefore we were not able to validate the generalizability of our model under 206 outbreaks dominated by VoCs. A modeling study suggested that differences in 207 population-level Ct values of samples from a symptom-based surveillance were more 208 likely to reflect changes in viral load trajectories instead of differences in 209 transmissibility across strains ${ }^{9,20}$. As such, if increased viral loads (i.e., lower Ct values) 210 occur with variant infections ${ }^{21}$, this may lead to decreased average Ct values when $R_{t}$ is 211 greater than 1. Leveraging information on factors affecting viral load levels (e.g., 
212 sequencing data) and regularly recalibrating the model will be necessary to update

213 exact values of parameters for accurate nowcasting of $R_{t}$ under more complex settings.

215 To summarize, in this analysis we applied a simplified method to incorporate the

216 population-level viral loads into real-time estimation of transmissibility for COVID-19.

217 We demonstrated that the Ct-based method could provide accurate nowcasting of $R_{t}$

218 potentially allowing capacity-constrained regions to track local outbreaks quantitatively

219 in a timely manner. Our method may need adaptions to different populations and the

220 evolving strains, mainly to recalibrate the absolute extent to which the population viral

221 loads correlate with COVID-19 transmissibility.

\section{Methods}

\section{Study settings}

225 Hong Kong was among the first places to identify COVID-19 cases globally, with its first

226 COVID-19 case detected in late January 20205,22. Cases were classified as "imported

227 cases”, "local cases epidemiologically linked with imported cases", "unlinked local cases”

228 and "local cases epidemiologically linked with local cases" according to their

229 epidemiological characteristics and location of infection. All suspected COVID-19 cases

230 were confirmed by RT-PCR in a local centralized public health laboratory. A laboratory-

231 confirmed COVID-19 case was defined as a local case if the case did not visit places

232 outside of Hong Kong in the 14 days before symptom onset (for symptomatic cases) or

233 confirmation (for asymptomatic cases); otherwise defined as imported cases.

235 By the time of analysis, four different waves of transmissions have occurred in Hong

236 Kong. In this study, we restricted our analyses to the third (July 2020 to August 2020) 
237 and fourth (November 2020 to March 2021) waves which were dominated by local

238 transmission, where 89\% were local cases. Since we were interested in the local

239 transmission of COVID-19, we only included local cases (unlinked local cases and local

240 cases epidemiologically linked with local cases) in our analyses. Given the stringent

241 border controls since July $2020^{23}$ and the extreme small number of local cases linked

242 with imported cases in Hong Kong $(<0.1 \%)$, we assumed that all unlinked local cases

243 were infected from other local cases. We did not include the first two waves (i.e.,

244 January to May 2020) as they were predominately imported cases and smaller clusters

245 linked to those imported cases $^{5,12}$.

In Hong Kong, local COVID-19 cases were generally detected from clinical diagnosis that targeted people with acute respiratory symptoms and from public health surveillance

249 that targeted population with predefined high risks of exposures (e.g., staff working at 250 healthcare centers; residents living in neighborhood with any lab-confirmed cases) by 251 health authorities ${ }^{11}$. Upon case confirmation, contact tracing was carried out based on 252 epidemiological information, with details described elsewhere ${ }^{24}$. Among 8869 local 253 COVID-19 cases confirmed between 1 July 2020 and 31 March 2021, 77\% were detected 254 symptomatic and 65\% were found with epidemiological links with other known cases; 255 across $23 \%$ of cases who were detected as asymptomatic, $81 \%$ of them were linked to 256 other local cases. As such, the surveillance of COVID-19 in Hong Kong is largely 257 symptom and contact-tracing based.

260 Data on viral load of COVID-19 cases. 
261 In Hong Kong, all confirmed COVID-19 cases (including asymptomatic cases) were

262 admitted to hospitals for isolation and standardized management, with their

263 hospitalization records stored in the data system managed by Hospital Authority (HA).

264 Results for SARS-CoV-2 RT-PCR tests (LightMix® Modular SARS-CoV-2 (COVID-19) E-

265 gene, TIB Molbiol/Roche, Berlin, Germany) ${ }^{25}$ were recorded as Ct values in the system.

266 The Ct value is the number of cycles needed to amplify the viral RNA in a specimen

267 where the reported fluorescent signal reaches a pre-defined level in RT-PCR assays.

268 Therefore, the Ct value is inversely associated with viral load, and could be used as a

269 semi-quantitative measurement for viral load. In our main analysis, we used Ct values to

270 measure viral load and analyzed the first recorded Ct value for each local case (which

271 was usually sampled on or one day before admission) during the study period.

272 Population viral load distributions were assessed by the date when samples were

273 collected.

Demographic and epidemiological information of confirmed COVID-19 cases.

We obtained demographic and epidemiological information from the Department of Health of the Government of Hong Kong, including age, date of symptom onset and case classification (i.e., local, imported and contacts of local or imported cases).

\section{$280 \quad$ Statistical methods}

281 Estimating incidence-based $R_{t}$.

282 We estimated the incidence-based $R_{t}$ for local cases using an extension of Cori et $283 \mathrm{al}^{4,26,27}$. Briefly, local COVID-19 cases confirmed on each day $t, Q_{1}(t)$ was used for 284 deconvolution to estimate the number of infections on each day $t, Y_{1}(t)^{28}$. We assumed 285 an average 5.2 days (SD 3.9) for the incubation period ${ }^{14}$ and an average 4.7 days (SD 3.2 
286 days, unpublished data) delay between illness onset to reporting empirically observed

287 in Hong Kong, which were used for deconvolution. In this framework, the daily local $R_{t}$ 288 (i.e., the incidence-based $R_{t}$ in our analysis) was the ratio between the number of new 289 local cases at time $t, Y_{1}(t)$, and the total infectiousness of cases at time $t$, given by $290 \sum_{k=1}^{t-1} Y_{1}(k) w_{L}(t-k)$, where $w_{L}(t-k)$ denote the probability of being infectious $t-k$

291 days after infections. The transmission was modeled by a Poisson process, and 292 therefore, we have

$$
Y_{1}(t) \sim \operatorname{Poisson}\left\{R_{t} \sum_{k=1}^{t-1} Y_{1}(k) w_{L}(t-k)\right\}
$$

$293 w_{L}(t-k)$ was estimated using the convolution of the incubation period (mean 5.2 days, 294 SD 3.9) ${ }^{14}$ and the infectiousness relative to onset ${ }^{2}$ (details described elsewhere ${ }^{4}$ ). To 295 fully utilize available case count information and to provide more timely $R_{t}$ estimates under the incidence-based method, we used the smoothing method described in Cori et $297 \mathrm{al}^{27}$ and calculated $R_{t}$ estimates over a time window of size $\tau=14$ ending at time $t$, 298 assuming that the transmissibility was constant over the time period $[t-\tau+1, t]$. We used a data-augmented Markov chain Monte Carlo algorithm to estimate the incidencebased $R_{t}$. To account for the uncertainty of other parameter such as the incubation period, we used an bootstrap approach in Salje et $\mathrm{al}^{29}$ to reconstruct 200 epidemic curves and perform estimation. After that we presented the mean, 2.5\% and $97.5 \%$ quantiles for those $200 R_{t}$ estimates for each day $t$. More details about incidence-based

$304 R_{t}$ estimation was described elsewhere ${ }^{4}$.

Temporal distribution of population-level Ct values.

307 We analyzed the first available Ct value record for each local COVID-19 case (i.e., $y_{j, t}, t$ is 308 the calendar date when the first sample was collected for individual $j$ ). To characterize 
309 the temporal distribution of population-level Ct values over the study period, we fitted a 310 generalized additive model (GAM) to the above-mentioned data over calendar time:

$$
y_{j, t}=\alpha_{0}+s(t)
$$

311 where $s(t)$ was the smooth function for date $t$ over study period. 95\% confidence

312 intervals (CIs) of the smoothed average daily Ct were derived from 500 bootstraps (Fig.

313 1b). In each bootstrap, we resampled from the data on cases' first available Ct values

314 and refitted the GAM. We did not include samples collected between 1 September 2020

315 to 31 October 2020 due to the small number of samples that were collected on each day.

317 To validate that the observed temporal variations in population-level Ct distribution

318 was not driven by variations in individual viral load trajectories, we estimated the Ct

319 value on the date of illness onset (Supplementary Fig. 2). We fitted a log-linear

320 regression of the first available $\mathrm{Ct}$ value for individual $j\left(y_{j}\right)$ on the time interval

321 between the individual's illness onset and first sample collection $\left(\delta_{j}\right)$ and age group $\left(a_{j}\right.$,

322 modelled as categorical, i.e., $0-18,19-64$ and $\geq 65$ years old):

$$
\ln \left(y_{j}\right)=\beta_{j}+\beta_{1} \delta_{j}+\beta_{2} a_{j}+\beta_{3} \delta_{j} a_{j} \quad \text { Eq.3 }
$$

323 where $\beta_{1}, \beta_{2}$ and $\beta_{3}$ are the estimated coefficients for the time interval between the 324 illness onset and first sample collection, age group and their interaction, respectively.

325 We then calculated the back-projected Ct value at illness onset by setting $\delta_{j}=0$. We 326 chose the log-linear model as the Akaike information criterion (AIC) indicated it 327 outperformed the linear model in terms of model fit ( -9177 and 38279 for the log328 linear and linear models, respectively).

330 We found that the skewness of Ct values at sampling was more variable (coefficient of 331 variation: 0.80 ) than the skewness of Ct values extrapolated at onset (coefficient of 
332 variation: 0.37) (Supplementary Fig. 2), suggesting that variations in individual viral

333 load trajectories may not be the major driver of the observed temporal variation in

334 population-level Ct distribution over our study period during which only the wild-type

335 SARS-CoV-2 strains have been circulating locally.

Incorporating Ct distributions into $R_{t}$ estimation (Ct-based $R_{t}$ ).

338 We used the mean $\left(\bar{x}_{t}\right)$ and skewness $\left(b_{t}\right)^{30}$ to measure the distribution of Ct values that 339 were sampled on date $t$ :

$$
\begin{gathered}
\bar{x}_{t}=\frac{1}{n_{t}} \sum_{i=1}^{n_{t}} y_{t, i} \\
b_{t}=\frac{\frac{1}{n_{t}} \sum_{i=1}^{n_{t}}\left(y_{t, i}-\bar{x}_{t}\right)^{3}}{\left[\frac{1}{n_{t}-1} \sum_{i=1}^{n_{t}}\left(y_{t, i}-\bar{x}_{t}\right)^{2}\right]^{\frac{3}{2}}}
\end{gathered}
$$

340 where $y_{t, i}$ represented the $i$ th $\left(i=1,2, \ldots, n_{t}\right)$ of the total $n_{t}$ Ct values that were sampled 341 on day t. 95\% CIs of the daily skewness $b_{t}$ were calculated from 500 bootstraps (Fig.

342 1c), with data on cases' first available Ct values resampled in each bootstrap to re343 calculate the daily skewness.

345 We first calculated the Spearman's rank correlation coefficient $(\rho)$ between daily Ct

346 distribution (i.e., daily mean and skewness) and the natural log-transformed incidence-

347 based $R_{t}$ (Supplementary Table 1$)$.

To determine the best fit model that characterized the association between daily $\mathrm{Ct}$

350 distribution and the incidence-based $R_{t}$, we compared AIC of a series of regression

351 models over the training period (i.e., 6 July 2020 to 31 August 2020), which used

352 different formats of dependent variable and measurements for predictive variables 
353 (Supplementary Table 3). We compared models that were fitted to linear scale and

354 natural log-transformed incidence-based $R_{t}$. We also assessed models that included

355 different combinations of measurements for daily Ct distributions, including mean,

356 median and skewness. We imputed the daily Ct distributions using the average of that

357 within the preceding 7 days when no samples were collected on that day. The model

358 fitted to natural log-transformed incidence-based $R_{t}\left(\ln \left(R_{t}\right)\right)$ on daily mean $\left(\bar{x}_{t}\right)$ and

359 skewness $\left(b_{t}\right)$ of Ct values was found with the lowest AIC and was used in our main

360 analyses (Supplementary Table 3):

$$
\ln \left(R_{t}\right)=\gamma_{0}+\gamma_{\bar{x}} \overline{x_{t}}+\gamma_{b} b_{t} \quad \text { Eq.6 }
$$

361 where $\gamma_{\bar{x}}$ and $\gamma_{b}$ were coefficients for daily mean and skewness of Ct values from the

362 regression model and were reported in Supplementary Table 2 after exponential

363 transformation.

365 To assess whether our results would be affected by the age distribution of cases who

366 were sampled on each day, we performed a sensitivity analysis by including the mean

367 age $\left(\overline{a_{t}}\right)$ of cases whose first sample were collected on day $t$ into the above-mentioned

368 main model (Eq. 6):

$$
\ln \left(R_{t}\right)=\gamma_{0}+\gamma_{\bar{x}} \overline{x_{t}}+\gamma_{b} b_{t}+\gamma_{\bar{a}} \overline{a_{t}}
$$

369 Results suggested similar predictions from models with and without considering cases' 370 age distribution (Supplementary Fig. 3).

372 Cross-validations of the model.

373 To validate the generalizability of this Ct-based method, we fitted the main model (Eq.

374 6) using data from an alternative training period, i.e., from 20 November 2020 to 31 
375 December 2020 (the initial stage of the fourth wave) (Supplementary Fig. 4,

376 Supplementary Table 2).

378 We further performed a 10-fold cross-validation by randomly splitting the data

379 between 6 July 2020 and 31 March 2021 into ten validation sets, after excluding days

380 when less than five available Ct samples were collected. For each validation, we held

381 one set as testing set, and trained the rest nine sets using the main model (Eq. 6). We

382 compared the consistency between the Ct- and incidence-based $R_{t}$ for the testing set by

383 calculating the proportion of days when the two estimates were simultaneously below

384 or above 1 (i.e., in the same direction) over total duration of each validation set. We also

385 assessed the prediction performance using the mean absolute error (MAE) for the Ct-

$386\left(E\left(R_{t}\right)\right)$ and incidence-based $R_{t}$ for each validation set:

$$
M A E=\frac{\sum_{d \in D}\left|\ln \left(E\left(R_{d}\right)\right)-\ln \left(R_{d}\right)\right|}{N_{D}}
$$

387 where $d$ is a given date in the validation set $D$ and $N_{D}$ is the number of days included in 388 each validation set. We found an average of 0.28 (ranging from 0.25 to 0.34 ) of the MAE 389 across ten validation sets, suggesting a good performance of our model predictions.

\section{Data availability}

392 All the data supporting the findings of this study are available and included in this 393 article and its supplementary information files.

\section{Code availability}

396 The source code will be available after publication in the GitHub repository 397 (https://github.com/vanialin/ct_rt_hk). 


\section{Acknowledgments}

399 We thank the Department of Health and Hospital Authority of the Food and Health

400 Bureau of the Government of Hong Kong for providing the data for the analysis. This

401 project was supported by the Health and Medical Research Fund, Food and Health

402 Bureau, Government of the Hong Kong Special Administrative Region (grant no.

403 COVID190118) and the Theme-based Research Scheme (Project No. T11-712/19-N) of

404 the Research Grants Council of the Hong Kong SAR Government.

\section{5}

406 Author contributions

407 All authors meet the ICMJE criteria for authorship. The study was conceived by BJC and 408 BY. YL, EHYL, JYTW, HSB, JKC, FH, HG and TKT prepared the data. BY and YL developed 409 the model. YL and BY conducted the data analyses. SC, DCA, STA, NHLH, TKT, PW, GML 410 and BJC interpreted the results. YL and BY wrote the first draft of the manuscript. All 411 authors provided critical review and revision of the text and approved the final version.

\section{Competing Interests Statement}

414 BJC consults for AstraZeneca, GSK, Moderna, Roche and Sanofi Pasteur. The authors 415 report no other potential conflicts of interest. 


\section{References}

4181 Gostic, K. M. et al. Practical considerations for measuring the effective reproductive number, Rt. PLOS Computational Biology 16, e1008409, doi:10.1371/journal.pcbi.1008409 (2020). He, X. et al. Temporal dynamics in viral shedding and transmissibility of COVID19. Nature Medicine 26, 672-675, doi:10.1038/s41591-020-0869-5 (2020). 267, doi:10.7326/M20-1495 (2020).

Tsang, T. K., Wu, P., Lau, E. H. Y. \& Cowling, B. J. Accounting for imported cases in estimating the time-varying reproductive number of COVID-19 in Hong Kong. The Journal of Infectious Diseases, doi:10.1093/infdis/jiab299 (2021). Real-time dashboard, <https://covid19.sph.hku.hk/> (2021). course. Science 373, eabi5273, doi:10.1126/science.abi5273 (2021). Ke, R. et al. Daily sampling of early SARS-CoV-2 infection reveals substantial heterogeneity in infectiousness. medRxiv, doi:10.1101/2021.07.12.21260208 (2021).

Wölfel, R. et al. Virological assessment of hospitalized patients with COVID-2019. Nature 581, 465-469, doi:10.1038/s41586-020-2196-x (2020).

Hay, J. A. et al. Estimating epidemiologic dynamics from cross-sectional viral load distributions. Science, eabh0635, doi:10.1126/science.abh0635 (2021). Tso, C. F., Garikipati, A., Green-Saxena, A., Mao, Q. \& Das, R. Correlation of Population SARS-CoV-2 Cycle Threshold Values to Local Disease Dynamics: 
Exploratory Observational Study. JMIR Public Health Surveill 7, e28265, doi:10.2196/28265 (2021).

11 Yang, B. et al. Universal Community Nucleic Acid Testing for COVID-19 in Hong Kong Reveals Insights into Transmission Dynamics. Research Square, doi:10.2139/ssrn.3844899 (2021).

12 Yang, B. et al. Changing Disparities in Coronavirus Disease 2019 (COVID-19) Burden in the Ethnically Homogeneous Population of Hong Kong Through Pandemic Waves: An Observational Study. Clinical Infectious Diseases, doi:10.1093/cid/ciab002 (2021).

$13 \mathrm{Gu}$, H. et al. SARS-CoV-2 under an elimination strategy in Hong Kong. medRxiv, 2021.2006.2019.21259169, doi:10.1101/2021.06.19.21259169 (2021).

14 Li, Q. et al. Early Transmission Dynamics in Wuhan, China, of Novel CoronavirusInfected Pneumonia. New England Journal of Medicine 382, 1199-1207, doi:10.1056/NEJMoa2001316 (2020).

15 Andriamandimby, S. F. et al. Cross-sectional cycle threshold values reflect epidemic dynamics of COVID-19 in Madagascar. medRxiv, 2021.2007.2006.21259473, doi:10.1101/2021.07.06.21259473 (2021).

16 Pitzer, V. E. et al. The Impact of Changes in Diagnostic Testing Practices on Estimates of COVID-19 Transmission in the United States. American Journal of Epidemiology, doi:10.1093/aje/kwab089 (2021).

17 Tsang, T. K. et al. Effect of changing case definitions for COVID-19 on the epidemic curve and transmission parameters in mainland China: a modelling study. Lancet Public Health 5, e289-e296, doi:10.1016/s2468-2667(20)30089-x (2020). 
18 Peccia, J. et al. Measurement of SARS-CoV-2 RNA in wastewater tracks community infection dynamics. Nature Biotechnology 38, 1164-1167, doi:10.1038/s41587-020-0684-z (2020).

19 Levine-Tiefenbrun, M. et al. Initial report of decreased SARS-CoV-2 viral load after inoculation with the BNT162b2 vaccine. Nature Medicine 27, 790-792, doi:10.1038/s41591-021-01316-7 (2021).

20 Hay, J. A., Kennedy-Shaffer, L. \& Mina, M. J. Viral loads observed under competing strain dynamics. medRxiv, 2021.2007.2027.21261224, doi:10.1101/2021.07.27.21261224 (2021).

21 Frampton, D. et al. Genomic characteristics and clinical effect of the emergent SARS-CoV-2 B.1.1.7 lineage in London, UK: a whole-genome sequencing and hospital-based cohort study. The Lancet Infectious Diseases, doi:10.1016/S1473$3099(21) 00170-5$.

22 Cowling, B. J. et al. Impact assessment of non-pharmaceutical interventions against coronavirus disease 2019 and influenza in Hong Kong: an observational study. The Lancet Public Health 5, e279-e288, doi:10.1016/S24682667(20)30090-6 (2020).

23 Yang, B. et al. The differential importation risks of COVID-19 from inbound travellers and the feasibility of targeted travel controls: A case study in Hong Kong. The Lancet Regional Health - Western Pacific 13, doi:10.1016/j.lanwpc.2021.100184 (2021).

24 Adam, D. C. et al. Clustering and superspreading potential of SARS-CoV-2 infections in Hong Kong. Nature Medicine 26, 1714-1719, doi:10.1038/s41591020-1092-0 (2020). 
25 Tsui, E. L. H. et al. Development of a data-driven COVID-19 prognostication tool to inform triage and step-down care for hospitalised patients in Hong Kong: a population-based cohort study. BMC Medical Informatics and Decision Making 20, 323, doi:10.1186/s12911-020-01338-0 (2020).

26 Abbott, S. et al. Estimating the time-varying reproduction number of SARS-CoV-2 using national and subnational case counts [version 2; peer review: 1 approved with reservations]. Wellcome Open Research 5, doi:10.12688/wellcomeopenres.16006.2 (2020).

27 Cori, A., Ferguson, N. M., Fraser, C. \& Cauchemez, S. A New Framework and Software to Estimate Time-Varying Reproduction Numbers During Epidemics. American Journal of Epidemiology 178, 1505-1512, doi:10.1093/aje/kwt133 (2013).

29 Salje, H. et al. Reconstruction of antibody dynamics and infection histories to evaluate dengue risk. Nature 557, 719-723, doi:10.1038/s41586-018-0157-4 (2018). Joanes, D. N. \& Gill, C. A. Comparing measures of sample skewness and kurtosis. Journal of the Royal Statistical Society: Series D (The Statistician) 47, 183-189, doi:https://doi.org/10.1111/1467-9884.00122 (1998). 


\section{Figure Legends}

514 Fig. 1 Correlations between temporal distribution of Ct values and transmission

515 dynamics of COVID-19 in Hong Kong. a, Local COVID-19 cases and the estimated

516 incidence-based $R_{t}$. Grey bars indicate the number of laboratory-confirmed local cases

517 by date of reporting. Black lines and shaded areas indicate the mean and $95 \%$ credible

518 intervals (CrIs) for incidence-based $R_{t}$. b, Ct distributions smoothed from a generalized

519 additive model (GAM). Dark grey bars indicate the number of sample collections.

520 Orange lines and shaded areas indicate the daily average and 95\% confidence intervals

521 (CIs) of Ct values that were estimated from a GAM (Eq. 2) over the study period in Hong

522 Kong. c, Daily skewness of Ct values over the study period. Blue dots represent the

523 observed daily Ct skewness and vertical lines represent 95\% CIs of daily Ct skewness

524 that were calculated from 500 bootstraps. d-e, Correlations between the daily

525 incidence-based $R_{t}$ and the daily mean Ct (d) or skewness (e). Boxes represent the

526 interquartile and median of the incidence-based $R_{t}$.

Fig. 2 Nowcast of the transmission dynamics of COVID-19 using Ct distribution. a,

529 Nowcasting $R_{t}$ using the Ct-based method over four representative weeks. Grey bars

530 represent the number of laboratory-confirmed local cases by date of reporting. Black

531 lines and shaded areas indicate the mean and 95\% CrIs for incidence-based $R_{t}$ (same

532 for $\mathbf{b}-\mathbf{c}$ ). Pink dots and vertical lines represent point estimates and $95 \%$ prediction

533 intervals for Ct-based $R_{t}$ estimated from Eq. 6 (same for b-c). b-c, Comparison of

534 incidence-based $R_{t}$ and Ct-based $R_{t}$ over the training period (July 2020-August 2020, b)

535 and the testing period (November 2020-March 2021, c). d, Distributions of incidence-

536 based $R_{t}$ under various intervals of Ct-based $R_{t}$. Boxes represent the interquartile and

537 median of incidence-based $R_{t}$ under the corresponding interval of Ct-based $R_{t}$ over

538 training (purple) and testing (pink) periods. 
541 Supplementary Figure 1. Daily distribution of Ct values by sampling date over the

542 study period. Dark grey vertical lines and red dots showed the interquartile and 543 median of Ct values sampled per day, while light grey vertical lines represented either 544 the minimum and maximum of all Ct values or the smallest and largest observed $\mathrm{Ct}$ values that are within the distance of 1.5 times the interquartile range of all Ct values on that day (i.e., whiskers). Orange lines and shaded areas represent the daily average and the $95 \% \mathrm{CI}$ of $\mathrm{Ct}$ values estimated from a GAM as in Eq. 2 (same as Fig. 1).

\section{Supplementary Figure 2. Bi-weekly distribution of Ct values by date of sampling} and of onset during the third (a) and fourth (b-c) wave of COVID-19 in Hong Kong. The Ct value shown by date of sampling was the actual value as sampled, whereas the $\mathrm{Ct}$ value shown by date of onset was the extrapolated value estimated from Eq. 3. The distribution of Ct values at sampling and at onset was illustrated in green and orange if during periods with increasing epidemic and in cyan and pink if during periods with decreasing epidemic. The skewness of Ct distribution at sampling $\left(b_{s}\right)$ and at onset $\left(b_{o}\right)$ over the biweekly period was shown at top-right of each panel with corresponding colors, while the exact time period was specified above each panel.

\section{Supplementary Figure 3. Consistent Ct-based $\boldsymbol{R}_{t}$ estimates before and after} adjusting for the age distribution of sampled cases. a-b, Comparison of daily mean Ct (a) and Ct skewness (b) under various incidence-based $R_{t}$ intervals during July 2020 - August 2020 (wave 3) and November 2020 - March 2021 (wave 4). Boxes show the interquartile and median of the corresponding Ct distribution derived from all samples (colored) and from samples of adult cases only (white) respectively. c) Comparison of Ct-based $R_{t}$ estimates before and after adjustment of age. Pink lines and shaded areas show the point estimates and 95\% prediction intervals of Ct-based $R_{t}$ estimated from the main model (Eq. 6), while blue dots and vertical lines show the point estimates and 95\% prediction intervals of Ct-based $R_{t}$ estimated after adjusting for the mean age of daily sampled cases (as in Eq. 7).

\section{Supplementary Figure 4. Validation of the $\mathrm{Ct}$-based model using an alternative}

572 training period. a-b, Comparison of incidence-based and Ct-based $R_{t}$ over the 573 alternative training (November-December 2020, a) and testing (July-August 2020, b) 
574 periods. Black lines and shaded areas indicate the mean and 95\% CrIs for incidence575 based $R_{t}$, while colored dots and vertical lines represent the point estimates and 95\% 576 prediction intervals of Ct-based $R_{t}$. c) Consistency between the incidence-based and Ct-

577 based $R_{t}$ over the alternative training (purple) and testing (pink) periods.

\section{Supplementary Figure 5. Performance of models fitted over various length and} timing of training periods. a-b, Performance of models fitted over different training periods with various durations and starting dates, over a) July-September 2020 and b) November 2020-January 2021. Color lines show the performance of models (measured by adjusted $\mathrm{R}$ square) fitted over training periods with a duration of 30-60 days respectively, with the starting date of the training period specified in the $\mathrm{x}$-axis. The grey dashed line in the background referred to adjusted $\mathrm{R}$ square being 0.7 , as a reference for comparison. c-d, Time period covered by the model with the largest adjusted R square over c) July-September 2020 and d) November 2020-January 2021. Grey and orange bars represent the number of laboratory-confirmed cases (by date of reporting) and of sampled collections (by date of sampling) respectively. Time periods used for fitting the model with the largest adjusted R square among all models fitted over that certain length of training period was indicated by corresponding colored

592 backgrounds (same color legend as in panel a). 


\section{Figures}
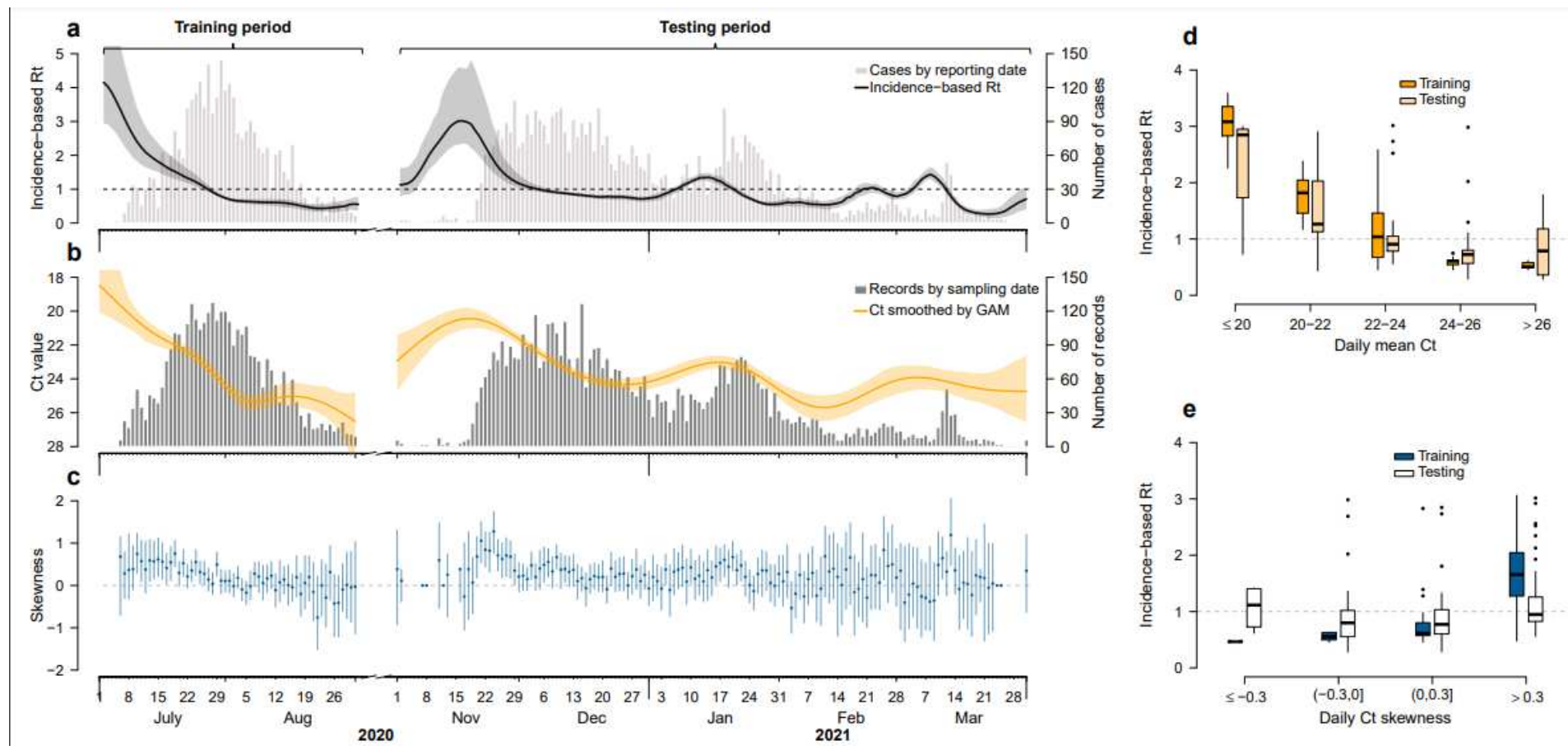

\section{Figure 1}

Correlations between temporal distribution of $\mathrm{Ct}$ values and transmission dynamics of COVID-19 in Hong Kong. a, Local COVID-19 cases and the estimated incidence-based R_t. Grey bars indicate the number of laboratory-confirmed local cases by date of reporting. Black lines and shaded areas indicate the mean and $95 \%$ credible intervals (Crls) for incidence-based R_t. b, Ct distributions smoothed from a generalized additive model (GAM). Dark grey bars indicate the number of sample collections. Orange lines and shaded areas indicate the daily average and $95 \%$ confidence intervals (Cls) of Ct values that were estimated from a GAM (Eq. 2) over the study period in Hong Kong. c, Daily skewness of Ct values over the study period. Blue dots represent the observed daily Ct skewness and vertical lines represent $95 \% \mathrm{Cls}$ of daily Ct skewness that were calculated from 500 bootstraps. d-e, Correlations between the daily incidence-based R_t and the daily mean Ct (d) or skewness (e). Boxes represent the interquartile and median of the incidence-based R_t. 


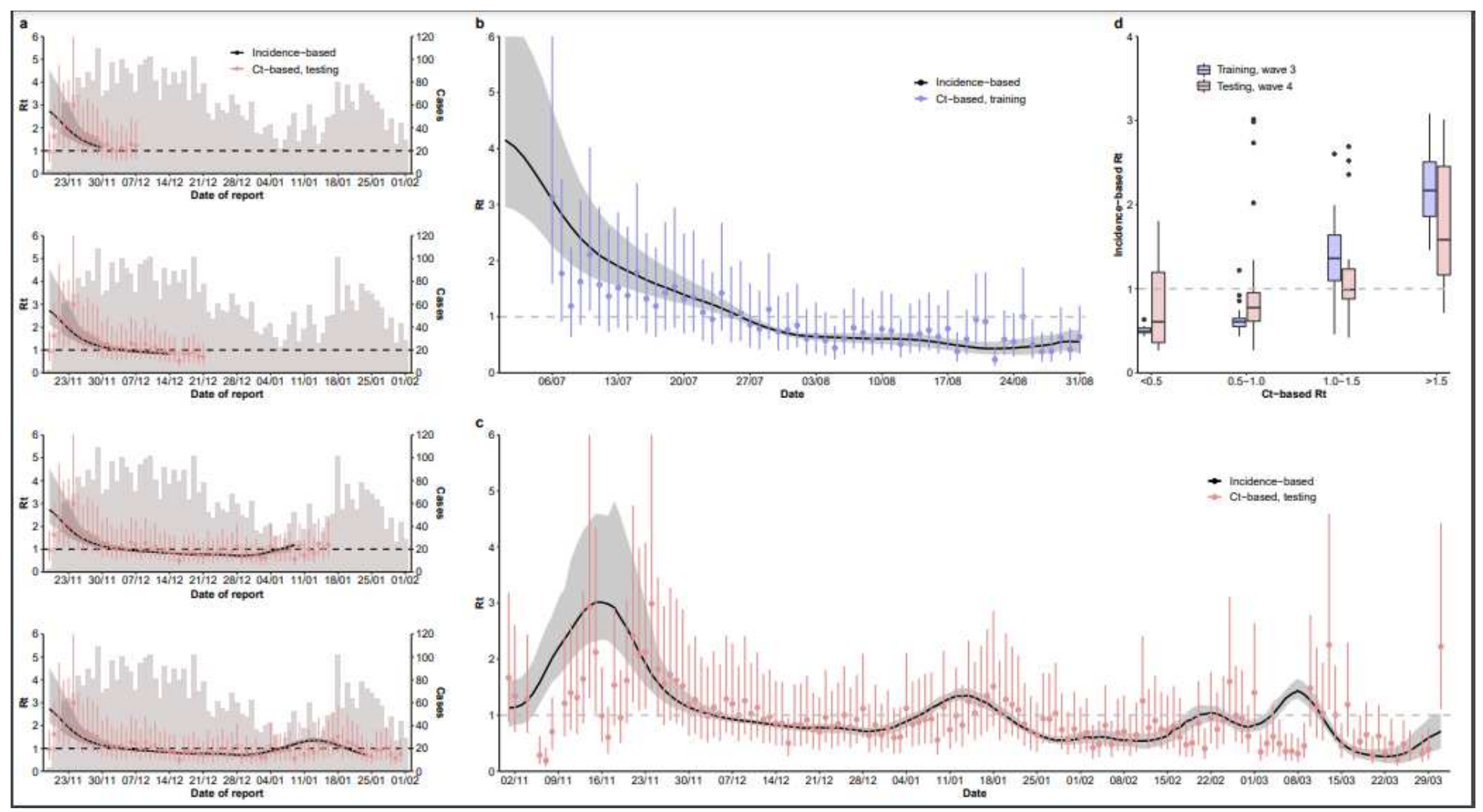

Figure 2

Nowcast of the transmission dynamics of COVID-19 using Ct distribution. a, Nowcasting R_t using the Ctbased method over four representative weeks. Grey bars represent the number of laboratory-confirmed local cases by date of reporting. Black lines and shaded areas indicate the mean and $95 \%$ Crls for incidence-based R_t (same for b-c). Pink dots and vertical lines represent point estimates and $95 \%$ prediction intervals for Ct-based R_t estimated from Eq. 6 (same for b-c). b-c, Comparison of incidencebased R_t and Ct-based R_t over the training period (July 2020-August 2020, b) and the testing period (November 2020-March 2021, c). d, Distributions of incidence-based R_t under various intervals of Ctbased R_t. Boxes represent the interquartile and median of incidence-based R_t under the corresponding interval of Ct-based R_t over training (purple) and testing (pink) periods.

\section{Supplementary Files}

This is a list of supplementary files associated with this preprint. Click to download.

- supplementary23aug.docx

- FigS1.pdf

- FigS2.pdf

- FigS3.pdf

- FigS4.pdf

- FigS5.pdf 